Revista Tecné, Episteme y Didaxis: TED. Año 2014, Número Extraordinario. ISSN Impreso: 0121-3814, ISSN web: 2323-0126 Memorias, Sexto Congreso Internacional sobre Formación de Profesores de Ciencias. 08 al 10 de octubre de 2014, Bogotá

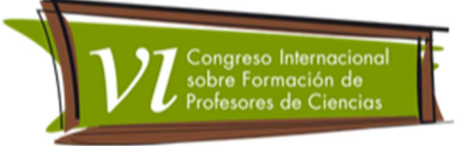

\title{
Influencia de la planeación docente en el desarrollo de habilidades cognitivo-lingüísticas
}

Briñez-Ortega, Edwin'; Quevedo-Acosta, Yovanny² y Aristizabal, Andrea'

Categoría 1. Reflexiones y experiencias desde la innovación en el aula.

\section{Resumen}

En esta ponencia se reflexiona sobre la aproximación hacia el desarrollo de las competencias argumentativas de los estudiantes en ciencias experimentales en el contexto escolar, como resultado de la planeación docente mediante una secuencia didáctica fundamentada en el constructivismo y relacionada con la dimensión comunicativa, para fortalecer el desarrollo progresivo de las habilidades cognitivo-lingüísticas de los estudiantes; en particular se fortalece una de las habilidades comunicativas primordiales en el lenguaje científico como es la descripción en los procesos de producción escrita.

\section{Palabras clave}

Habilidades cognitivo-lingüísticas, texto descriptivo, eficiencia docente.

\section{Introducción}

En los procesos de alfabetización científica se pretende reestructurar las concepciones alternativas y adoptar posturas más cercanas al pensamiento científico; en esta transición es indispensable cuestionar el concepto de eficiencia docente y migrar su significado tradicionalista relacionado con los resultados o el rendimiento académico a uno más cercano al constructivismo, una concepción que asuma como docente eficiente a un profesional en didáctica y pedagogía, propositivo y capaz de generar efectos positivos en sus estudiantes (Aristizabal y García, 2012).

La eficiencia docente, en general, se relaciona con las estrategias utilizadas para mejorar el rendimiento académico, teniendo en cuenta que está ligado exclusivamente a la evaluación de los contenidos. Lejos de esta posición, es posible asumir la eficiencia de un docente como los efectos que él pueda generar en sus estudiantes acerca de la concepción del mundo. La eficiencia

\footnotetext{
${ }^{1}$ Facultad de Ciencias y Educación, Universidad Distrital Francisco José de Caldas, Bogotá D.C. embrinezo@correo.udistrital.edu.co, andrea_aristizabal@hotmail.com. 2 Departamento de Química, Facultad de Ciencias, Universidad Nacional de Colombia-sede Bogotá D.C. eyquevedoa@unal.edu.co.
} 
Revista Tecné, Episteme y Didaxis: TED. Año 2014, Número Extraordinario. ISSN Impreso: 0121-3814, ISSN web: 2323-0126 Memorias, Sexto Congreso Internacional sobre Formación de Profesores de Ciencias. 08 al 10 de octubre de 2014, Bogotá

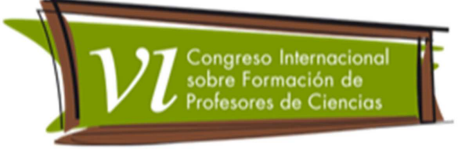

del docente no puede ser concebida como el éxito en la mecanización, memorización o adaptación de procedimientos, sino todo lo contrario, un docente eficiente no debe permitir que sus estudiantes mecanicen procesos, memoricen formulas, entre otros (Rodríguez, Núñez, Valle, Blas y Rosario, 2009). Un docente eficiente debe incentivar la curiosidad, el pensamiento crítico y permitir la comprensión del mundo desde la visión científica, pues, en últimas es lo que se procura en el proceso de alfabetización científica (Flores, 2001).

En este sentido, la eficiencia docente en la enseñanza de las ciencias en general y de la química en particular, se relaciona con el proceso y la trayectoria de la formación de personas reflexivas y críticas, que de acuerdo con García y Pinilla (2007) serían generadoras de estrategias para la solución de los problemas que se presentan en la sociedad; objetivo estrechamente relacionado con la construcción del aprendizaje, a través de las habilidades cognitivas y comunicativas de los estudiantes.

Desde esta perspectiva, en esta ponencia se establece que la planeación docente a través de la construcción e implementación de una secuencia didáctica en un contexto escolar, basada en la comunicación escrita, el trabajo práctico de laboratorio y el enfoque constructivista, puede ser efectiva para el proceso de aprendizaje de las ciencias, el desarrollo de las competencias científicas y las habilidades cognitivo-lingüísticas de los estudiantes, específicamente en la producción de textos descriptivos como proceso de aproximación progresiva al nivel de pensamiento argumentativo y reflexivo.

\section{Desarrollo}

La educación científica actual debe fomentar el pensamiento crítico, el desarrollo y uso de habilidades y talentos que contribuyan al mejoramiento de las condiciones sociales, en ese sentido la enseñanza de las ciencias naturales proporciona un ambiente ideal para el desarrollo de habilidades de pensamiento y destrezas significativas en la vida de los estudiantes (Lemke, 2006). Así, hablar en ciencias, en el aula, no se limita al uso de términos técnicos, por el contrario implica observar, describir, comparar, clasificar, analizar, discutir, cuestionar, decidir, concluir, enseñar y resolver problemas en la escuela y en la vida cotidiana (Lemke, 1997).

De acuerdo a lo anterior, es razonable que no se aprende ciencia solo de la experiencia directa sino que se hace necesario conocer cómo se comunican las experiencias en el discurso científico. Para este efecto, es pertinente que el docente, teniendo en cuenta los intereses de los estudiantes, construya 
Revista Tecné, Episteme y Didaxis: TED. Año 2014, Número Extraordinario. ISSN Impreso: 0121-3814, ISSN web: 2323-0126 Memorias, Sexto Congreso Internacional sobre Formación de Profesores de Ciencias. 08 al 10 de octubre de 2014, Bogotá

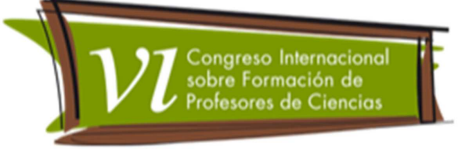

estrategias fundamentadas en el trabajo práctico, específicamente el que corresponde al de laboratorio pues es en este espacio donde experimentan, observan, controlan variables, formulan hipótesis, interpretan datos, elaboran modelos y evalúan el proceso (habilidades cognitivas). También, con actividades de laboratorio, se da lugar al trabajo en equipo, a la comunicación de ideas desde lo descriptivo pasando por lo explicativo hasta lo argumentativo (Sanmartí, 2008).

En ese orden de ideas se puede afirmar que la inclusión de las prácticas de laboratorio en la planeación docente implica en los estudiantes un aumento considerable de las destrezas procedimentales, y también colabora en una aproximación del desarrollo de las competencias argumentativas como lo plantean Briñez-Ortega, Quevedo-Acosta y Aristizabal (2012), que observaron un nivel de conceptualización estructurado, preciso, coherente y organizado de las ideas de los estudiantes en el proceso de producción de los textos; además evidenciaron que las actividades de laboratorio planteadas en las estrategias de enseñanza influyeron en la fluidez y elocuencia que tuvieron las estudiantes en la comunicación escrita de sus conocimientos.

De acuerdo con lo anterior y con Jorba (2000), en la producción de textos, los estudiantes llevan a cabo un proceso de verbalización que resulta favorable para establecer relaciones entre lo que se ha aprendido y los nuevos contenidos presentados por el docente, permitiendo de manera significativa que el aprendizaje permanezca y sea útil. Esto es posible gracias al proceso de comprensión que antecede la producción de textos escritos, es decir, que de acuerdo al tipo de texto que se pretenda elaborar pueden estimularse diversos sucesos cognitivos. Estos procesos de relación cognitiva y comunicativa se denominan habilidades cognitivo-lingüísticas.

De acuerdo con esto, al docente le corresponde reflexionar sobre el modo más adecuado para desarrollar el pensamiento argumentativo de los estudiantes, y una posible forma es a través de la habilidad para describir, porque a partir de esta se recopilan las principales características de los fenómenos, que no siguen una relación explícita pero que involucran las competencias científicas como la observación, la comparación y la identificación de lo esencial que, según Jorba, Gómez, y Prat (2000), hacen viable la elaboración textual con una terminología técnica donde la complejidad de la descripción varía si el objeto es concreto o abstracto, simple o complejo, presencial o no presencial y si los fenómenos son o no observables y directamente perceptibles. 
Revista Tecné, Episteme y Didaxis: TED. Año 2014, Número Extraordinario. ISSN Impreso: 0121-3814, ISSN web: 2323-0126 Memorias, Sexto Congreso Internacional sobre Formación de Profesores de Ciencias. 08 al 10 de octubre de 2014, Bogotá

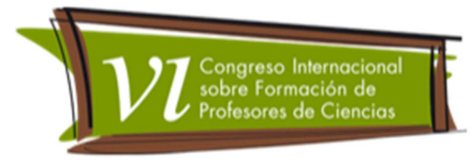

Por esta razón, resulta de gran importancia el diseño de unidades didácticas, que permiten concretar el qué y el cómo se va a enseñar, de acuerdo con los objetivos de aprendizaje que se establezcan entre el docente y los estudiantes. A partir de estos, se seleccionan y secuencian unos contenidos y actividades interrelacionados partiendo de lo más concreto a lo más abstracto, y que tienen una trascendencia social, como se ilustra en Quevedo et al (2013) mediante una Unidad Didáctica fundamentada en comunicación, que a través del concepto de célula se secuencian las estrategias que permitirían a los estudiantes aproximarse al desarrollo de competencias argumentativas, por ejemplo acerca del aporte social del químico Louis Pasteur a través del proceso científico, en higiene (pasteurización) y salud pública (vacuna), en la industria (producción de vino y seda) y en la academia (microbiología).

Para evaluar la efectividad de la planeación docente, es conveniente la propuesta de Quevedo-Acosta et al (2012), en el diseño y aplicación de un cuadro de valoración, considerando unos niveles y unas categorías de valoración teniendo en cuenta los criterios de evaluación de textos explicativos propuestos por Jorba (2000) y algunos elementos básicos de contenido (conceptos), estilo (estructura argumentativa) y presentación de un texto científico como son precisión y coherencia (Parra, 2001), que permiten analizar cualquier texto de carácter descriptivo de cualquier nivel de escolaridad o área del conocimiento.

El diseño de cuadros de valoración facilita la interpretación y el manejo de los datos, de la tendencia del desarrollo de las habilidades cognitivo-lingüísticas del grupo de estudiantes y también de manera individual. Por ejemplo podría asignarse una valoración de: 1, 2 ó 3 que corresponden a los niveles básico, intermedio y estructurado de un escrito descriptivo de acuerdo con la presencia o ausencia de las características propias de ese tipo de texto para el análisis estadístico.

Así, para la autoevaluación y autorregulación del docente, la eficiencia de la planeación de la secuencia didáctica se evidencia a través de la organización de las ideas de los estudiantes moldeadas en un texto escrito para poder expresar cómo sucede un hecho, de forma coherente, empleando un lenguaje científico escolar y desde su estructura conceptual propiamente construida, (Jorba, 1997).

De esta manera, se concluye que el diseño de unidades didácticas fundamentadas en comunicación científica es útil como estrategia de investigación e innovación en torno al lenguaje científico escolar, también es un modo de autoevaluación y autorregulación del desarrollo profesional 
Revista Tecné, Episteme y Didaxis: TED. Año 2014, Número Extraordinario. ISSN Impreso: 0121-3814, ISSN web: 2323-0126 Memorias, Sexto Congreso Internacional sobre Formación de Profesores de Ciencias. 08 al 10 de octubre de 2014, Bogotá

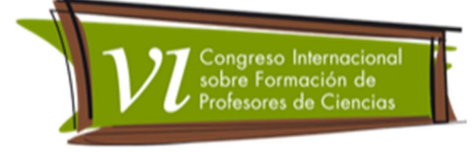

docente y de la eficiencia de la formación pedagógica de los estudiantes, asimismo proporciona elementos importantes que permiten dar el salto del docente repetidor de procedimientos al docente creativo e innovador.

Finalmente, el lenguaje es un medio indispensable para poder desarrollar el pensamiento, por ese motivo el docente eficiente promueve el desarrollo de habilidades cognitivo-lingüísticas a través de actividades prácticas orientadas por procesos comunicativos. Además, el diseño de unidades didácticas fundamentadas en comunicación es útil como estrategia de trabajo, y se plantea como sugerencia que sería importante y significativo extender la orientación de las unidades en la dimensión comunicativa a más de un área del conocimiento.

\section{Referencias Bibliográficas}

Aristizabal, A. y García, A. (2012). Construcción de la identidad profesional docente: ¿posibilidad o utopía?. Revista EDUCYT, 126-138.

Briñez-Ortega, E., Quevedo-Acosta, Y. y Aristizabal, A. (2012). Influencia de los trabajos prácticos de laboratorio en el desarrollo de habilidades cognitivo-lingüísticas. En: III Congreso Nacional EducyT y del II Congreso Iberoamericano CllEC. Pasto: Universidad de Nariño.

Campaner, G. y De Longhi, A. (2007). La argumentación en Educación Ambiental. Una estrategia didáctica para la escuela media. Revista Electrónica de Enseñanza de las Ciencias, 6: 442-456.

Flores, M. (2001). El Factor humano en la docencia de educación secundaria: un estudio de la eficacia docente y el estrés a lo largo de la carrera profesional. Tesis doctoral. Universitat Autónoma de Barcelona, España.

García, A. y Pinilla, J. (2006). Análisis cualitativo y comprensivo de resultados en la evaluación de la comprensión en ciencias de los niños y niñas de los grados 5 y 9‥ Bogotá: DIDAQUIM.

García, A. y Pinilla, J. (2007). Orientaciones curriculares para el campo de ciencia y tecnología. Bogotá: Secretaría de Educación.

Garton, (2001). Interacción social y desarrollo del lenguaje y la cognición. Barcelona: Paidós. 
Revista Tecné, Episteme y Didaxis: TED. Año 2014, Número Extraordinario. ISSN Impreso: 0121-3814, ISSN web: 2323-0126 Memorias, Sexto Congreso Internacional sobre Formación de Profesores de Ciencias. 08 al 10 de octubre de 2014, Bogotá

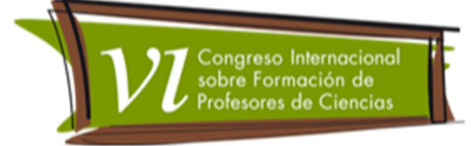

Izquierdo, M., Sanmartí, N. y Espinet, M. (1999). Fundamentación y diseño de las prácticas escolares de ciencias. Enseñanza de las ciencias, 17: 45-59.

Jorba, J. y Bisbal, Ester. (1997). La regulación y autorregulación de los aprendizajes. Madrid: Editorial Síntesis.

Jorba, J., Gómez, I. y Prat, Á. (2000). Hablar y Escribir para Aprender: uso del lenguaje en situación de enseñanza-aprendizaje desde las áreas curriculares. Madrid: Síntesis.

Lemke, J. (1997). Aprender a hablar ciencia: Lenguaje, aprendizaje y valores. Barcelona: Paidós.

Lemke, J. (2006). Investigar para el futuro de la educación Científica: nuevas formas de aprender, nuevas formas de vivir. Enseñanza De Las Ciencias, 24: $5-12$.

Martínez, F. y Parra, J. (2009). Desarrollo de habilidades cognitivo lingüísticas (describir, resumir, explicar) empleando una metodología de resolución de problemas. Tecné, Episteme y Didaxis, 1238- 1244.

Parra, M. (2001). Cómo se produce el texto escrito: teoría y práctica. Bogotá: Aula abierta.

Quevedo-Acosta, Y., Briñez-Ortega, E. y García, A. (2012). Elaboración de textos descriptivos como estrategia para el desarrollo de habilidades cognitivo-lingüísticas. Inédito.

Quevedo-Acosta, Y., Briñez-Ortega, E. y Aristizabal, A. (2013). ¿Qué es la vida y cómo está organizada?. En: Rojas Valdés, R., Manrique, F., Quintanilla, M. (Eds). Enseñanza de las ciencias naturales en las primeras edades. Su contribución a la promoción de competencias de pensamiento científico (vol. 7, pp 171-183). Santiago de Chile: Laboratorio GRECIA.

Rodríguez, S., Núñez, J., Valle, A., Blas, R. y Rosario, P. (2009). Auto-eficacia docente, motivación del profesor y estrategias de enseñanza. Escritos de Psicología, 3: 1-7.

Sánchez, J. (2006). Saber Escribir. Madrid: Instituto Cervantes. 
Revista Tecné, Episteme y Didaxis: TED. Año 2014, Número Extraordinario. ISSN Impreso: 0121-3814, ISSN web: 2323-0126 Memorias, Sexto Congreso Internacional sobre Formación de Profesores de Ciencias. 08 al 10 de octubre de 2014, Bogotá

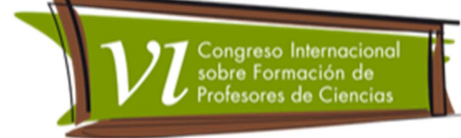

Sanmartí, N. (2008). La unidad didáctica en el paradigma constructivista. Barcelona: Universidad Autónoma de Barcelona. 